Arch. Tierz., Dummerstorf 45 (2002) 4, 359-373

Aus dem Institut für Tierzucht und Tierverhalten der Bundesforschungsanstalt für Landwirtschaft Braunschweig

MARTIN STEINHARDT und HANS - HERMANN THIELSCHER

\title{
Herzfrequenz, Aktivitäts- und Ruhezeiten sowie Wachstumsleistung von in Gruppen mit Tränkeautomatenfütterung gehaltenen Milchrindkälbern während der Aufzucht im Winter und Frühjahr
}

\begin{abstract}
Summary
Title of the paper: Heart rate, activity and resting times and growth in group reared feeder-fed dairy calves reared at transition from winter to spring

To characterize the food entrainable oscillator and/or light entrainable oscillator determined rhythmicity of physiological variables of feeder-fed dairy calves reared at transition from winter to spring (35 German Holstein Friesian, 2 German Red Pied, 17 male and 20 female) long term heart rate recordings were taken at 5, 15, 40 and 60 days of age using Polar Sport Tester, from which the number and duration of activity (ZDA) and rest periods (ZDR) and the total activity (GZA) and rest times (GZR) could be established. For these periods characteristic heart rate values were calculated (HFA and HFR) and they were analysed for daytime periods of three hours duration at different life ages. HF of feeder-fed dairy calves showed substantial interindividual variation and a high individual specificity. Mean HFA and HFR were significantly smaller at 15 and 40 days then at 5 and 60 days of age. HFA and HFR showed a high correlation and the mean values of HFA and HFR were allways significantly different. More profound increases of HF (HFA-HFR) could be observed at feeding and service times. Individual level of HFA and HFR changed regularly with daytime, but mean values were significantly different between daytime periods only at some age points. Deviation of HFA and HFR from the mean of the individual daytime heart rate recording followed a circadian rhythm that became more obvious with advanced development and adaptation of the animals in case of HFA and HFR. For the means of the deviations of HFA and HFR significant differences between the daytime periods could be found. The mean ZDA and the quotient GZA/GZR were greater at 40 and 60 days of age then at 5 and 15 days of age. ZDA and ZDR showed great variation and presented rhythmic changes during the course of the day. The means of ZDA and ZDR were significantly different between the daytime periods of three hours duration at the age points excluding that of ZDA for 15 days of age. Results show the establishment and the prevalence of a circadian rhythm of heart rate at the early growth period in feeder-fed dairy calves reared at transition from winter to spring and how this was superimposed by feeding activity around the programmed daytimes of feed access at the start of rearing.
\end{abstract}

Key Words: characteristic heart rate values, activity and resting times, diurnal rhythm, feeder - fed dairy calves, life age, seasonal effects

\section{Zusammenfassung}

Um die Rhythmizität physiologischer Variablen von Milchrindkälbern unter dem Einfluss des food-entrainableOscillators und des light-entrainable Oscillators im Verlaufe der frühen Wachstumsperiode zu charakterisieren, wurden während der Aufzucht im Übergang vom Winter zum Frühjahr an Kälbern (35 Deutsche Holstein Friesian, 2 Deutsche Rotbunte, 17 männliche, 20 weibliche) vor (5 Lebenstage, LT) und während der Gruppenhaltung am Tränkeautomaten (15, 40 und 60 LT) Langzeitmessungen der Herzfrequenz (HF) mit dem Polar Sport Tester vorgenommen. Mit Hilfe der HF-Kurven konnten Anzahl und Dauer von Aktivitäts- (ZDA) und Ruheperioden (ZDR), die tägliche Gesamtzeit der Aktivität (GZA) und der Ruhe (GZR) ermittelt, HF-Kennwerte für diese (HFA und HFR) errechnet und für Tageszeitbereiche von 3 Std. an den Alterspunkten analysiert werden. HF wies eine beträchtliche interindividuelle Variation und eine hohe Individualspezifität auf. HF war bei 15 LT und 40 LT signifikant kleiner als bei 5 LT und 60 LT. Herzfrequenzen der Aktivitäts- (HFA) und der Ruhezeiten (HFR) wiesen hohe Korrelationen auf, und die Mittelwerte von HFA und HFR unterschieden sich sicher. Größere Steigerungen von HF (HFA-HFR) waren häufiger an den Tränkezeiten zu beobachten. Das individuelle Niveau von HFA und HFR änderte sich an den Tageszeiten, und die Mittelwerte unterschieden sich zwischen den Tageszeitbereichen an einigen Alterspunkten sicher. Abweichungen der HFA und der HFR vom 
individuellen Mittelwert der Tagesaufzeichnung wiesen eine circadiane Periodik auf, die mit fortschreitender Entwicklung und Adaptation der Tiere bei HFA und HFR deutlicher hervortrat. Für die mittleren Abweichungen von HFA und HFR konnten zwischen den Tageszeitbereichen signifikante Unterschiede gefunden werden. Die mittleren ZDA und der Quotient GZA/GZR waren bei 40 und 60 LT größer als bei 5 und 15 LT. ZDA und ZDR wiesen eine große Variation und rhythmische Änderungen im Tagesverlauf auf. Die Mittelwerte von ZDA und ZDR waren zwischen den Tageszeitbereichen an den Alterspunkten mit Ausnahme von ZDA bei 15 LT signifikant verschieden. Die Untersuchungsbefunde zeigen, wie sich im Verlaufe von Wachstum und Adaptation beim Übergang vom Winter in das Frühjahr bei Kälbern ein circadianer Rhythmus der HF ausgeprägt hatte und vorherrschend wurde und wie dieser durch die mit der Nahrungsaufnahme verbundene Aktivierung im Beginn der Aufzucht überlagert wurde.

Schlüsselwörter: Herzfrequenzkennwerte, Aktivitäts- und Ruhezeite, diurnaler Rhythmus, Milchrindkälber, Tränkeautomatenfütterung, Altersabhängigkeit, Jahreszeit

1.

\section{Einleitung}

Die Rhythmizität der Funktionen und physiologischer Variablen ist hinsichtlich der Entwicklungsqualität der Tiere und der Optimierung ihrer Leistungsfähigkeit sowie der tiergerechten Haltung und des Wohlbefindens von Nutztieren von Interesse. Die mit der Nahrungsaufnahme verbundene Rhythmik physiologischer Variablen während der frühen Wachstumsperiode und ihre Beziehung zum Lichtrhythmus ist bei Kälbern kaum untersucht worden. Die Food-anticipatory activity (FAA) wird für den Output eines Oscillators gehalten, dessen Phase und Periode durch mit dem täglichen Fütterungsereignis verbundene Korrelate kontrolliert werden (MISTLBERGER, 1994). Das Modell postuliert einen durch Nahrung kontrollierten Oscillator (food entrainable oscillator, FEO) der gekoppelt ist an, aber separat existiert von einem übergeordneten Licht kontrollierten Oscillator (light entrainable oscillator, LEO). Über die Wirkung von FEO und LEO in Abhängigkeit von Entwicklungsqualität, Alter und Anpassung an spezifische Haltungsbedingungen bei Nutztieren ist wenig bekannt. Unter Bedingungen unbegrenzter Nahrungsverfügbarkeit wie bei Saugkälbern der Mutterkuhhaltung (STEINHARDT und THIELSCHER, 2001a; STEINHARDT, 2001) und vorübergehend begrenzter Nahrungsverfügbarkeit für die Tiere wie bei den am Tränkeautomaten aufgezogenen Milchrindkälbern könnten sich in den Jahreszeiten und bei deren Übergängen unterschiedliche Effekte von FEO und LEO auf die Periodizität physiologischer Variablen ergeben. Bei sich verkürzender Tageslichtzeit im Übergang vom Herbst zum Winter können die durch den Betriebsablauf vorhandenen Zeitgeber und Reizwirkungen einen größeren Einfluss auf rhythmische Funktionsabläufe bei Kälbern haben, während mit der Zunahme der Tageslichtzeit im Frühjahr Tageslichtwirkungen stärker werden.

Die Herzfrequenz (HF) in den Aktivitäts- und Ruhezeiten (HFA und HFR) der Saugkälber der Mutterkuhhaltung wies während der Winterstallhaltung mit Dämmerungsbeleuchtung bei kontinuierlicher Registrierung infradiane Schwingungen auf, die eine circadiane Periodik überlagerten (STEINHARDT und THIELSCHER, 2000c, 2001). Bei künstlich aufgezogenen Milchrindkälbern nehmen die Tierbetreuung und technische Maßnahmen je nach Haltungs- und Tränkesystem einen größeren Zeitraum ein. Dieser Aufwand ändert sich in Abhängigkeit von der Geburtenfrequenz, und es wird auch die Häufigkeit der Änderungen der Gruppenzusammensetzung beeinflußt. Die Anzahl der Kälber pro Gruppe nimmt gegen Ende der Kalbeperiode im allgemeinen ab, und der gesamte Betreuungsaufwand für die Aufzuchtkälber verringert sich. 
Im vorliegenden Untersuchungsansatz wurde die Rhythmizität physiologischer Variablen bei Kälbern während der Aufzucht unter gebräuchlichen Betriebsbedingungen in der Zeit des Überganges vom Winter zum Frühjahr charakterisiert. Folgende Fragen waren von Interesse: (1) Welche Rhythmik weist die HF von Milchrindkälbern bei der Aufzucht im Winter und Frühjahr auf? (2) Wie ist die Aktivitätsperiodik der Kälber während der Aufzucht am Tränkeautomaten bei kontinuierlicher Registrierung zu charakterisieren? (3) Welche Änderungen sind an der Aktivitätsperiodik und der HF mit dem Alter und der Adaptation der Tiere festzustellen? (4) Lassen sich Beziehungen zwischen der Dauer der Aktivitäts- und der Ruheperioden und der HF und deren Änderungen nachweisen?

Untersuchte Tiere, Haltungsbedingungen: Kälber der Milchrindherde des Institutes für Tierzucht und Tierverhalten (Deutsche Holstein Friesian, DHF; Deutsche Rotbunte, DRB), die in der Zeit vom 19.12.97 bis 26.04.98 geboren sind (insgesamt 35 DHF und 2 DRB; 17 männliche und 20 weibliche Kälber) wurden untersucht. Von zwei Zwillingspaaren (weiblich, männlich und männlich, männlich) waren jeweils ein männliches Tier in den ersten Lebenstagen verendet. Die verbliebenen Zwillingstiere sind in die Untersuchung einbezogen worden. Nach der Geburt blieben die Kälber bis zum Alter von 5 bis 7 Lebenstagen (LT) in einer Gruppenbox mit Stroheinstreu im Abkalbebereich des Stallgebäudes (Laufstall), in welchem die Milchrinder gehalten wurden. Die Kälber erhielten mehrmals Kolostrum der Mutter (07.00 Uhr und 16.00 Uhr, 1 bis 2 l pro Angebot), danach gepoolte Frischmilch aus einem Eimer mit Nuckel. Die Tiere erhielten 5 ml Myofer 200 (Hoechst) pro Tier über die Milch verabreicht. In Gruppenhaltung (2,5 $\mathrm{m}^{2}$ pro Tier bei voller Besetzung) mit Tränkeautomatenfütterung (Tränkeautomat der Fa. Förster, Festzeitsystem, Tränkeschema: 1 Woche 6 l; 1 Woche $6 \mathrm{l}$ auf $8 \mathrm{l}$ ansteigend; 4 Wochen 8 l; 6 Wochen $8 \mathrm{l}$ auf $2 \mathrm{l}$ abfallend; 1 Woche $2 \mathrm{l}$ pro Tag Milchaustauscher (MAT, 125 g pro Liter Wasser) verblieben die Kälber 6 Wochen als Einstallungsgruppe und danach 6 Wochen in einer benachbarten Box als Ausstallungsgruppe. Die MAT-Verfügbarkeit war auf 4 Zeitpunkte pro Tag (03.00 Uhr, 08.00 Uhr, 15.00 Uhr und 20.00 Uhr) beschränkt, pro Abruf konnten 2 l MAT erreicht werden, und die Sperrzeit nach Abruf betrug 120 min. Die Tränkestation war in der Nacht beleuchtet. Eine Lichterkette war von 03.00 bis 05.00 Uhr, von 08.00 bis 10.00 Uhr, von 15.00 bis 17.00 Uhr und von 20.00 bis 22.00 Uhr angeschaltet. Die Fenster des Aufzuchtstalles waren mit Windschutznetzen versehen, durch welche Tageslichtwirkungen möglich waren. Pelletiertes Kraftfutter konnte über einen Automaten ansteigend bis maximal $3 \mathrm{~kg}$ pro Tag abgerufen werden. Gleichzeitig wurden Heu und Silage angeboten, und es bestand die Möglichkeit der Wasseraufnahme aus Selbsttränken und der Kochsalzaufnahme an Lecksteinen. Die Messungen wurden bei einem Alter der Kälber von 5, 15, 40 und 60 LT vorgenommen (auch als Alterspunkte 1 bis 4 bezeichnet) und begannen nach der Fütterung. Die Servicezeit war für 10.00 bis 12.00 Uhr festgelegt. Wägungen der Kälber wurden an spezifischen Alterspunkten wie Geburt, Einstallung in die Gruppenhaltung, Umstallung in eine andere Gruppenhaltung und Ausstallung vorgenommen. Aus diesen Messungen ist die Wachstumsrate der Kälber errechnet worden (Tab. 1).

Untersuchungsablauf, Messungen: HF wurde mit dem Polar Sport Tester (Herzfrequenz Computer von Polar Electro OY, Kempele, Finnland) festgestellt. Das Gerät be- 
steht aus 3 Teilen: Empfänger, Sender und Brustgurt. Der Empfänger von der Größe einer Armbanduhr ermöglicht die Speicherung der HF in Intervallen von 5, 15 und 60 sec und die Einstellung von Grenzwerten sowie Alarmzeichen. Die Aufzeichnung der HF erfolgte in 60 sec-Intervallen über 24 Std. Die HF-Kurven wurden mit einem speziellen Programm bearbeitet und für die Aktivitäts- und Ruheperioden im Tagesverlauf HF-Kennwerte (HFA und HFR) errechnet. Die Steigerung der HF (HFA-HFR) ist im Tagesverlauf (Abb. 2) und in Beziehung zu den Aktivitätsperioden geprüft worden. Für jedes Tier ist ein Mittelwert der Messung für HFA und HFR ermittelt worden. Die HF-Werte der Aktivitäts- und Ruheperioden im Tagesverlauf ließen sich als Abweichungen von den individuellen Mittelwerten der Messung für HFA bzw. HFR (DIHFA und DIHFR) darstellen (Abb. 3, Tab. 4), um die Periodik erkennbar zu machen. Aus den HF-Kurven konnten außerdem Beginn und Dauer der Aktivitäts- (ZDA) und der Ruheperioden (ZDR) und die gesamte tägliche Aktivitäts- (GZA) und Ruhezeit (GZR) ermittelt werden. Beginn und Dauer der Aktivitäts- und Ruheperioden sind 8 Tageszeitbereichen (TZB) von jeweils 3 Std. zugeordnet worden, die die Grundlage für Berechnungen waren. Durch permanente Videoaufzeichnungen konnten die mit Hilfe der Herzfrequenzkurve ermittelten Aktivitäts- und Ruheperioden der Tiere verifiziert werden.

Tabelle 1

Körpermasse, zugebildete Körpermasse und Wachstumsrate der Milchrindkälber an verschiedenen Alterspunkten, Mittelwerte und Standardabweichung (Body weight, accumulated body weight and growth rate of dairy calves at different life ages, Mean values and Standard deviation)

\begin{tabular}{l|l|l|l|l|l}
\hline & $9 \mathrm{LT} *$ & $52 \mathrm{LT}$ & $79 \mathrm{LT}$ & $94 \mathrm{LT}$ & $101 \mathrm{LT}$ \\
\hline Körpermasse & 46,9 & 70,1 & 96,1 & 110,6 & 118,3 \\
$(\mathrm{~kg})$ & 4,3 & 8,3 & 10,2 & 11,3 & 11,8 \\
\hline Zugebildete Körpermasse & 2,7 & 25,9 & 51,9 & 66,5 & 74,2 \\
(kg) & 3,2 & 6,2 & 9,1 & 10,3 & 10,8 \\
\hline Tägliche Zunahme & 0,288 & 0,503 & 0,653 & 0,712 & 0,739 \\
(g/d) & 0,343 & 0,115 & 0,11 & 0,107 & 0,106 \\
\hline
\end{tabular}

* Lebenstage

Bearbeitung der Ergebnisse, statistische Methoden: Die Auswertung fand unter folgenden Gesichtspunkten statt: (1) Prüfung der HF und der ZDA und ZDR sowie GZA und GZR zwischen den Alterspunkten, (2) Prüfung der Beziehungen von HFA und HFR in den Tageszeitbereichen, an den Alterspunkten und zwischen den Alterspunkten, (3) Prüfung der HF, der Steigerung von HF (HFA-HFR) und der Abweichungen der HF vom individuellen Mittelwert der Tagesaufzeichnungen sowie der ZDA und ZDR zwischen den Tageszeitbereichen an den Alterspunkten, (4) Prüfung der Beziehung von GZA und GZR an den Alterspunkten und (5) Prüfung der Beziehung der genannten Meßgrößen zu den Wachstumskriterien. Die Bearbeitung der Ergebnisse wurde mit Statistikpaketen wie PC-Statistik von Topsoft Hannover und Sigma Stat von Jandel Scientific Software vorgenommen und die Korrelations- und Regressionsrechnung sowie die Varianzanalyse (ANOVA und ANOVA for repeated measures) angewandt. Wenn Normalverteilung und Gleichheit der Varianzen nicht gegeben war, wurde die Kruskal-Wallis Varianzanalyse und die Friedman Varianzanalyse für wiederholte Messungen auf der Basis von Rangplätzen genutzt. Multiple Mittelwertprüfungen wurden mit dem Bonferroni t-Test, nach der Student-Newman-Keuls-Methode oder nach der Methode von Dunn, Mittelwertprüfungen zweier Gruppen mit dem tTest und Wilcoxon-Test vorgenommen. Die Irrtumswahrscheinlichkeiten sind in den 
Tabellen und Abbildungen angegeben und, wenn nicht ausdrücklich erwähnt, mit 5 \% angenommen worden.

\section{Ergebnisse}

\section{Körpermasse, Wachstum}

Das mittlere Geburtsgewicht der Kälber betrug 44,2 \pm 4,9 kg (32 bis 52 kg). In den ersten 10 LT wies die Wachstumsrate eine große Variation auf, die sich bis zum Alter von $52 \mathrm{LT}$ verringerte und dann bis $101 \mathrm{LT}$ etwa gleich blieb (Tab. 1). Die tägliche Zunahme (TZN) bei 9 LT unterschied sich gegen TZN 101 LT $(p<0,001)$, TZN bei 52 LT unterschied sich gegen TZN bei 79 LT, 94 LT und 101 LT $(p<0,001)$ und TZN bei 79 LT unterschied sich gegen TZN bei 94 LT $(p=0,024)$ und $101 \mathrm{LT}(p=0,001)$. Die Variation der KM und der zugebildeten KM nahm bis zum Alter von 79 LT zu (Tab. 1). Ein Einfluss der Geburtsmasse auf die KM der Kälber ließ sich bis zum Alter von 101 LT nachweisen.

\section{Herzfrequenz, Aktivitäts- und Ruhezeiten der Milchrindkälber an verschiedenen Alterspunkten}

HFA und HFR sowie auch die Steigerung (HFA-HFR) änderten sich im Mittel bis zum Alter der Kälber von 60 LT geringgradig (Tab. 2). Bemerkenswert waren die größeren Streuungen der meisten Variablen (Abb. 1, Tab. 2). Die Mittelwerte und die Häufigkeitsverteilungen von HFA und HFR unterschieden sich zwischen den Alterspunkten in einigen Fällen signifikant, diejenigen der Steigerung von HF (HFA-HFR) nicht (Abb. 4). HFA und HFR unterschieden sich an den Alterspunkten stets signifikant. Zwischen HFA und HFR ließen sich enge Korrelationen $(p<0,0001)$ nachweisen.

Tabelle 2

Herzfrequenz der Aktivitäts- und der Ruheperioden, Steigerung der Herzfrequenz, Zeitdauer der Aktivitäts- und der Ruheperioden sowie Gesamtzeit der Aktivität und der Ruhe und deren Quotient von Milchrindkälbern an 4 Alterspunkten, Mittelwerte und Standardabweichungen (Heart rate (bpm) at activity- and at resting periods, increase in heart rate , time (min) of activity- and of resting periods and total daily time in minutes of activity and of resting and their quotient in dairy calves at four life ages, Mean values and Standard deviation)

\begin{tabular}{l|l|l|l|l}
\hline & $\begin{array}{l}5 \mathrm{LT}^{*} \\
(210) * *\end{array}$ & $\begin{array}{l}15 \mathrm{LT} \\
(252)\end{array}$ & $\begin{array}{l}40 \mathrm{LT} \\
(239)\end{array}$ & $\begin{array}{l}60 \mathrm{LT} \\
(248)\end{array}$ \\
\hline $\begin{array}{l}\text { Herzfrequenz der Aktivitäsperioden } \\
\text { (HS/min) }\end{array}$ & 107 & 102 & 102 & 107 \\
11 & 15 & 17 & 11 \\
\hline Herzfrequenz der Ruheperioden & 91 & 85 & 85 & 91 \\
(HS/min) & 14 & 13 & 16 & 10 \\
\hline Steigerung der Herzfrequenz & 16,3 & 16,2 & 17,0 & 16,6 \\
(HS/min) & 8,8 & 8,2 & 8,0 & 7,1 \\
\hline Zeitdauer der Aktivitätsperioden & 41 & 45 & 77 & 79 \\
(min) & 36 & 32 & 68 & 62 \\
\hline Zeitdauer der Ruheperioden & 81 & 78 & 83 & 82 \\
(min) & 48 & 49 & 56 & 56 \\
\hline Gesamtzeit der Aktivität am Tag & 452 & 490 & 664 & 695 \\
(min) & 118 & 120 & 128 & 128 \\
\hline Gesamtzeit der Ruhe am Tag & 918 & 821 & 737 & 705 \\
(min) & 101 & 140 & 128 & 130 \\
\hline Quotient Gesamtzeit der Aktivität/ & 0,51 & 0,63 & 0,96 & 1,05 \\
Gesamtzeit der Ruhe & 0,19 & 0,24 & 0,34 & 0,39 \\
\hline * Lebenstage;* Anzahl der Messungen & & &
\end{tabular}


Abb. 1: Polar Plots der Herzfrequenz (Herzschläge/min) (linker Teil) und der Zeitdauer (min) (rechter Teil) der Aktivitäts- (volle Zeichen ) und Ruheperioden (leere Zeichen) der Milchrindkälber im Alter von 5, 15, 40 und 60 Lebenstagen (von oben nach unten), Einzelwerte (Polar Plots of heart rate (bpm) (left part) and time periods in minutes (right part) of activity (full symbols) and of resting times (empty symbols) of dairy calves at 5, 15, 40 and 60 days of life age (top to bottom), single values)
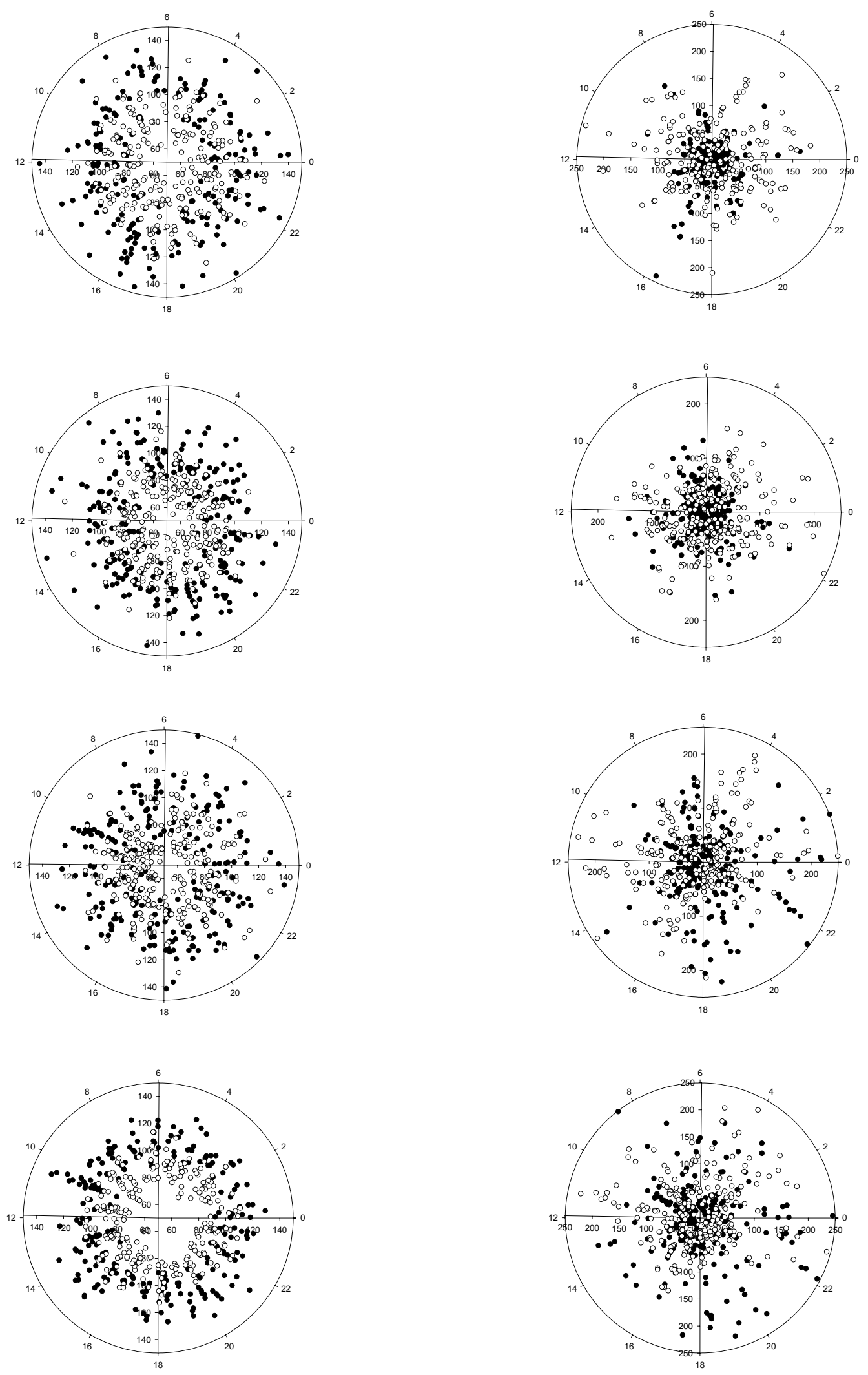
Abb. 2: Steigerung der Herzfrequenz (HFA-HFR) von Milchrindkälbern im Tagesverlauf an 4 Alterspunkten, Einzelwerte (Increase of heart rate (HFA-HFR) in dairy calves with the course of a day at four age points, single values)
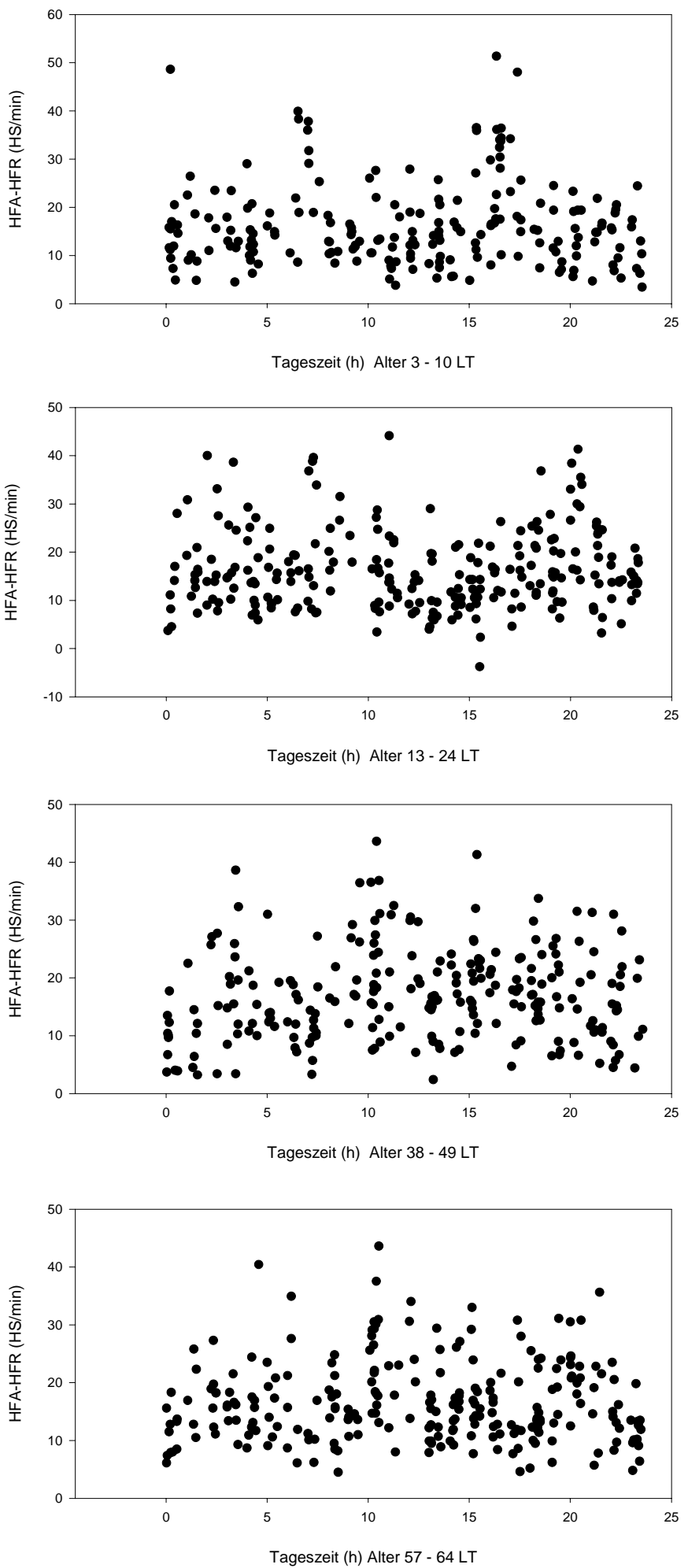
Ein Einfluss des Geschlechtes auf HFA konnte bei 5 LT, 15 LT und 60 LT, ein solcher auf HFR bei 15 LT und 60 LT und auf die Steigerung von HF (HFA-HFR) bei 5 LT und $40 \mathrm{LT}$ festgestellt werden. HFA und HFR lagen bei weiblichen Tieren im Alter von 5 LT und 60 LT höher und im Alter von 15 LT niedriger als bei männlichen Tieren. Die Steigerung von HF war bei weiblichen Tieren im Alter von 5 LT und 40 LT größer als bei männlichen Tieren. Bei Kälbern im Alter von 5 LT, 15 LT und 60 LT ließ sich eine signifikante negative Korrelation von HFA und HFR mit dem Alter der Tiere nachweisen.

ZDA und ZDR wiesen an allen Alterspunkten keine Normalverteilung und eine große Variation auf (Abb. 1 und 4, Tab. 2). Die Mittelwerte und die Verteilungen derselben unterschieden sich zwischen einigen Alterspunkten sicher (Tab. 4). Auffällig waren die größeren mittleren ZDA der Kälber im Alter von 40 LT und 60 LT gegenüber 5 LT und 15 LT (Tab. 2). Zwischen GZA und GZR der Kälber ließ sich eine negative Korrelation nachweisen, die an den Alterspunkten $40 \mathrm{LT}$ und $60 \mathrm{LT}$ enger $(\mathrm{r}=0,962$ und $r=0,962)$ als bei $5 \mathrm{LT}$ und $15 \mathrm{LT}(\mathrm{r}=0,811$ und $\mathrm{r}=0,687)$ war.

\section{Periodik von Herzfrequenz, Aktivität und Ruhe}

Häufungen größerer HF und größerer Steigerungen der HF an den Tränkepunkten (07.00 und 16.00 Uhr) bei Kälbern im Alter von 5 LT und an den Abrufzeitpunkten des MAT (03.00, 08.00, 15.00 und 20.00 Uhr) bei Kälbern im Alter von 15, 40 und 60 LT sowie in der Servicezeit sind an Abbildung 1 und 2 zu erkennen. Die mittleren HFA und die mittleren HFR der Tageszeitbereiche unterschieden sich an einigen, diejenigen von HFA-HFR sowie DIHFA und DIHFR an allen Alterspunkten sicher (Abb. 1 bis 3, Tab. 3). Die Verteilung der Abweichungen der HF vom individuellen Mittelwert der Tagesaufzeichnung deutete im Tagesverlauf bei HFA und HFR in den ersten Lebenstagen eine circadiane Periodik an (Abb. 3). Die Schwingungen wurden deutlicher bei einem Alter der Kälber von 15 LT und 40 LT. Stärkere Einflüsse durch die Milch- bzw. MAT-Aufnahme sind sowohl an der Zunahme von HFA als auch besonders an derjenigen von HFR zu sehen. Steigerungen von HFA sind im Beginn der Aufzucht am Tränkeautomaten während der Abrufzeiten größer als am Ende der Ernährungsperiode mit Flüssignahrung. An allen Untersuchungspunkten sind größere Steigerungen von HFA während der Servicezeit festzustellen gewesen. Die Schwingungen von HFA im Tagesverlauf verringerten sich, als die Kälber in die benachbarte Entwöhnungsgruppe gekommen waren. Bei HFR waren die Schwingungen im Tagesverlauf bis zum Alter von 15 LT in stärkerem Maße durch die Milch- bzw. MAT-Aufnahme beeinflußt. Bei einem Alter der Kälber von 40 und 60 LT trat eine circadiane Schwingung deutlicher hervor.

Häufungen längerer ZDA und ZDR und deren Änderungen mit dem Alter der Kälber sind an Abbildung 1 zu sehen. Länger andauernde ZDA aber auch ZDR sind bei Kälbern im Alter von 40 und $60 \mathrm{LT}$ in der Tageszeit zwischen $12.00 \mathrm{Uhr}$ und $00.00 \mathrm{Uhr}$ festzustellen gewesen (Abb. 1). Die Mittelwerte von ZDA und ZDR waren zwischen den Tageszeitbereichen an den Alterspunkten mit Ausnahme von ZDA bei 15 LT signifikant verschieden (Abb. 1 und 3, Tab. 3). Bemerkenswert waren die Verteilungen von ZDA und ZDR der Kälber im Alter von 5 LT (Abb. 4) und die Änderungen der Häufigkeiten von ZDA in der Klasse 20 min und diejenigen von ZDR in den Klassen 40, 60, 80 und 100 min mit dem Alter der Tiere (Abb. 4). Für GZA konnte eine Beziehung bei dem Alter von 60 LT und 15 LT $(p=0,01)$ und für GZR eine solche bei 60 
LT und 5 LT ( $(p=0,05)$ sowie $60 \mathrm{LT}$ und $15 \mathrm{LT}(\mathrm{p}=0,019)$ festgestellt werden. Der Quotient GZA/GZR wies zwischen 60 LT und 15 LT eine Korrelation auf ( $p=0,006)$.

Abb. 3: Abweichung der Herzfrequenz (Herzschläge/min) vom Mittelwert der individuellen Tagesaufzeichnung während der Aktivitäts- (linker Teil) und der Ruheperioden (rechter Teil) von Milchrindkälbern an 4 Alterspunkten, Einzelwerte (Deviation of heart rate (bpm) from the mean value of activity (left part) and of resting periods (right part) from the individual dayly record at four age points, single values)
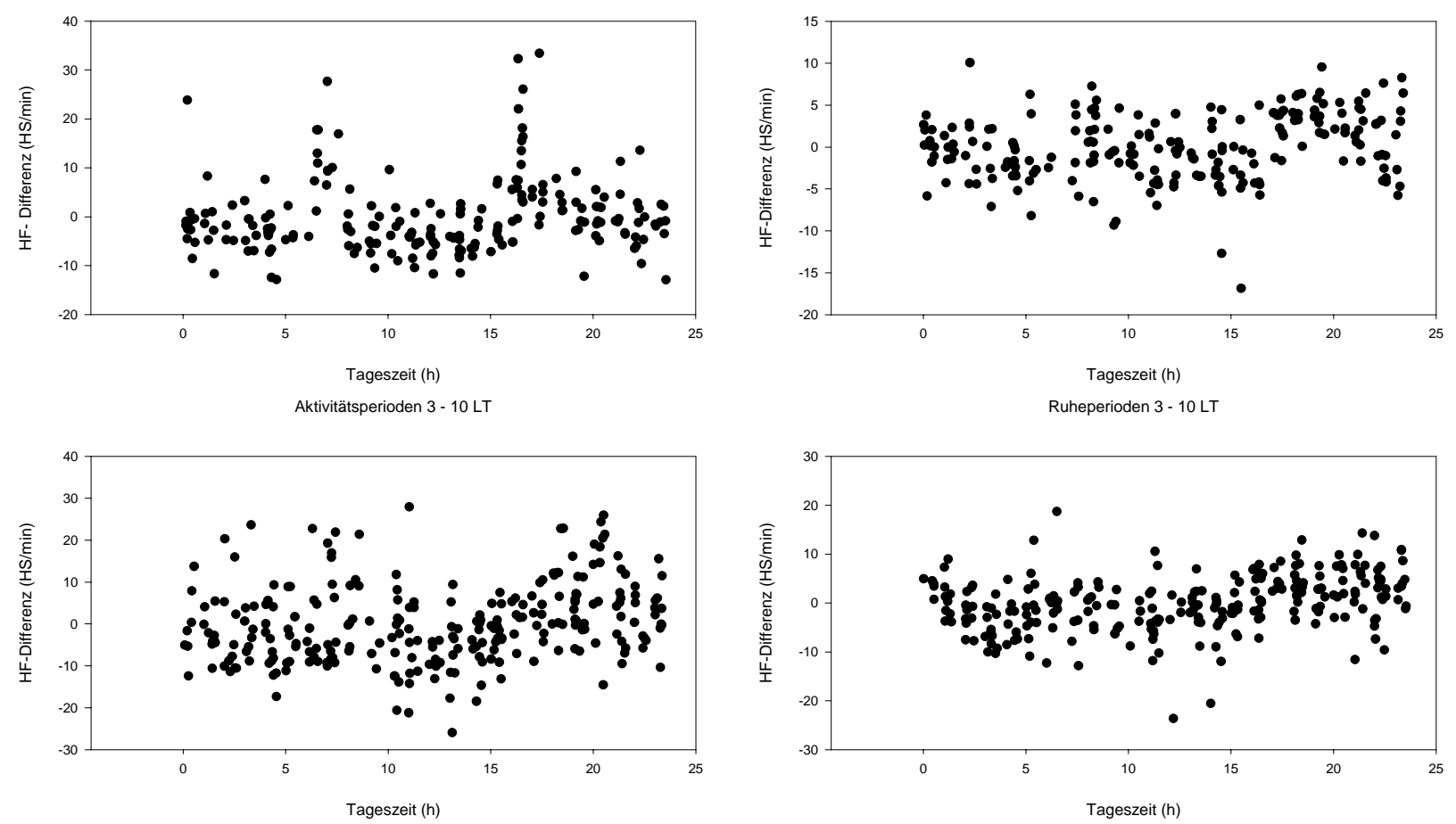

Aktivitätsperioden 13 - 24 LT

Ruheperioden 13 - 24 LT
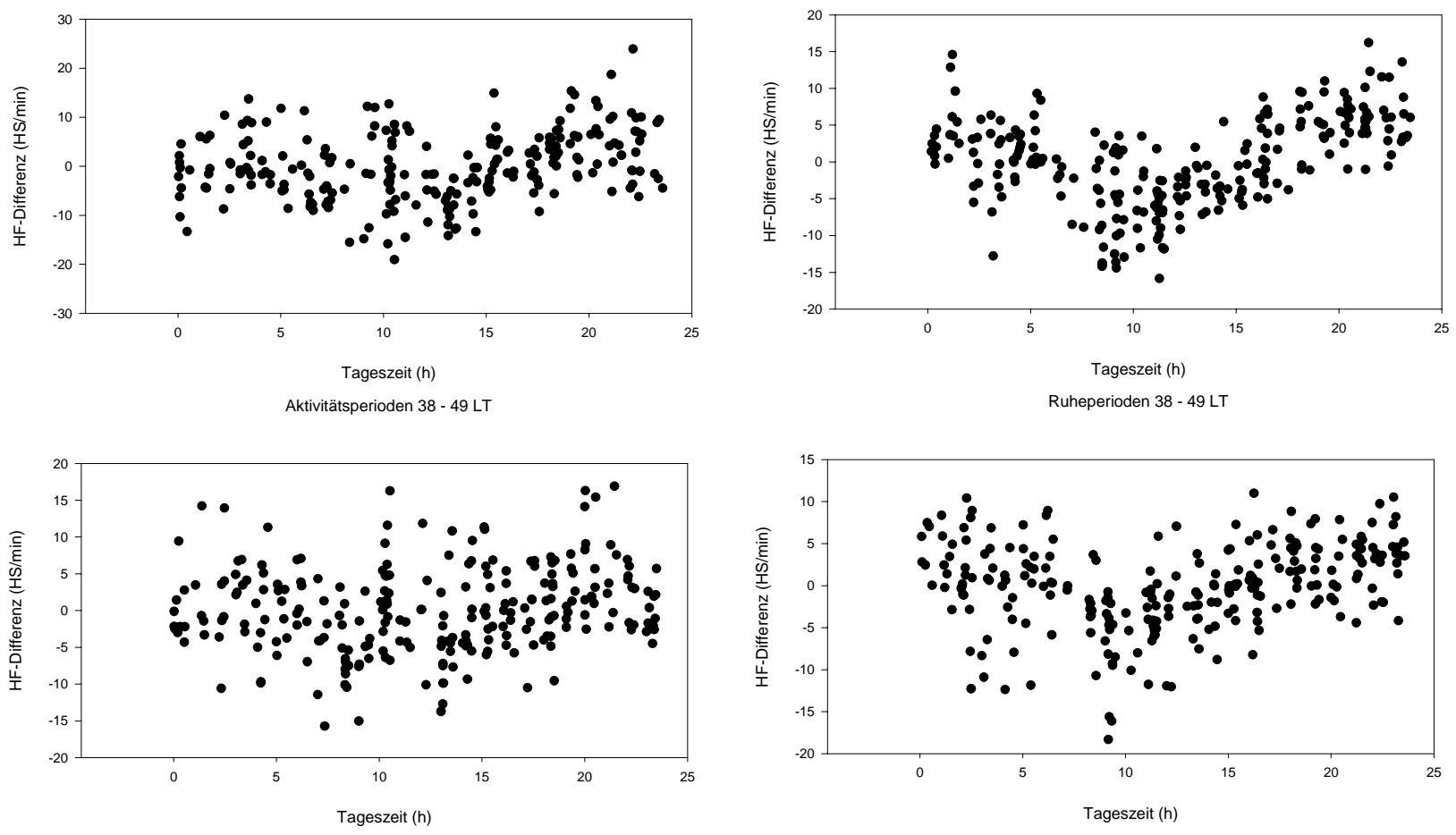

Aktivitätsperioden 57 - 64 LT 
Tabelle 3

Übersicht zu den Mittelwertunterschieden der Variablen zwischen den Tageszeitbereichen von 3 Std. Dauer an 4 Alterspunkten (Review of mean value differences of the variables between day time periods of three houres duration at four age points)

\begin{tabular}{l|l|l|l|l}
\hline & $5 \mathrm{LT}^{*}$ & $15 \mathrm{LT}$ & $40 \mathrm{LT}$ & $60 \mathrm{LT}$ \\
\hline Herzfrequenz der Aktivitätsperioden & & $\mathrm{P}<0,001$ & & \\
\hline Herzfrequenz der Ruheperioden & & & $\mathrm{P}<0,001$ & $\mathrm{P}<0,001$ \\
\hline Steigerung der Herzfrequenz & $\mathrm{P}<0,001$ & $\mathrm{P}<0,001$ & $\mathrm{P}<0,001$ & $\mathrm{P}=0,016$ \\
\hline $\begin{array}{l}\text { Abweichung der Herzfrequenz } \\
\text { während der Aktivitätsperioden }\end{array}$ & $\mathrm{P}<0,001$ & $\mathrm{P}<0,001$ & $\mathrm{P}<0,001$ & $\mathrm{P}<0,001$ \\
\hline $\begin{array}{l}\text { Abweichung der Herzfrequenz } \\
\text { während der Ruheperioden }\end{array}$ & $\mathrm{P}<0,001$ & $\mathrm{P}<0,001$ & $\mathrm{P}<0,001$ & $\mathrm{P}<0,001$ \\
\hline Zeitdauer der Aktivitätsperioden & $\mathrm{P}=0,006$ & & $\mathrm{P}<0,001$ & $\mathrm{P}=0,02$ \\
\hline Zeitdauer der Ruheperioden & $\mathrm{P}<0,001$ & $\mathrm{P}<0,001$ & $\mathrm{P}<0,001$ & $\mathrm{P}<0,001$ \\
\hline
\end{tabular}

* Lebenstage

Tabelle 4

Übersicht zu den Unterschieden der Mittelwerte (oberer Teil) und der Häufigkeitsverteilungen (unterer Teil) der Zeitdauer (min) der Aktivitäts- und der Ruheperioden sowie der Gesamtzeit der Aktivität und der Ruhe und deren Quotient von Milchrindkälbern zwischen den Alterspunkten 1 bis 4 (Review of differences of the mean values (upper part) and of the frequency distributions (lower part) of the time (min) of activity and resting periods and of total daily activity und resting and their quotient between the age points 1 to 4 )

\begin{tabular}{|c|c|c|c|}
\hline & $15 \mathrm{LT}^{*}$ & $40 \mathrm{LT}$ & $60 \mathrm{LT}$ \\
\hline $\begin{array}{l}\text { LT } \text { Zeitdauer der Aktivitätsperioden } \\
\text { Zeitdauer der Ruheperioden } \\
\text { Gesamtzeit der Aktivität } \\
\text { Gesamtzeit der Ruhe } \\
\text { Quotient Gesamtzeit der Aktivität/ } \\
\quad \text { Gesamtzeit der Ruhe }\end{array}$ & $P=0,014$ & $\begin{array}{l}\mathrm{P}<0,0001 \\
\mathrm{P}<0,0001 \\
\mathrm{P}<0,0001 \\
\mathrm{P}<0,0001\end{array}$ & $\begin{array}{l}\mathrm{P}<0,0001 \\
\mathrm{P}<0,0001 \\
\mathrm{P}<0,0001 \\
\mathrm{P}<0,0001\end{array}$ \\
\hline $\begin{array}{l}15 \text { LT Zeitdauer der Aktivitätsperioden } \\
\text { Zeitdauer der Ruheperioden } \\
\text { Gesamtzeit der Aktivität } \\
\text { Gesamtzeit der Ruhe } \\
\text { Quotient Gesamtzeit der Aktivität/ } \\
\text { Gesamtzeit der Ruhe }\end{array}$ & & $\begin{array}{l}\mathrm{P}<0,0001 \\
\mathrm{P}<0,0001 \\
\mathrm{P}=0,028 \\
\mathrm{P}<0,0001\end{array}$ & $\begin{array}{l}\mathrm{P}<0,0001 \\
\mathrm{P}<0,0001 \\
\mathrm{P}=0,003 \\
\mathrm{P}<0,0001\end{array}$ \\
\hline $\begin{array}{l}5 \text { LT } \text { Zeitdauer der Aktivitätsperioden } \\
\text { Zeitdauer der Ruheperioden }\end{array}$ & $\begin{array}{l}\mathrm{P}=0,0105 \\
\mathrm{P}=0,0225\end{array}$ & $\mathrm{P}<0,0001$ & $\begin{array}{l}\mathrm{P}<0,0001 \\
\mathrm{P}=0,0003\end{array}$ \\
\hline $\begin{array}{l}15 \text { LT Zeitdauer der Aktivitätsperioden } \\
\text { Zeitdauer der Ruheperioden }\end{array}$ & & $\mathrm{P}<0,0001$ & $\mathrm{P}<0,0001$ \\
\hline
\end{tabular}

* Lebenstage

Individuelle Variation und Individualspezifität der Herzfrequenzkennwerte

Die beträchtliche individuelle Variation von HF ist an Tabelle 2 sowie an den Abbildungen 1 und $4 \mathrm{zu}$ erkennen. Mit Hilfe der Korrelationsrechnung wurde geprüft, in welchem Maße HF unter verschiedenen Bedingungen in individualspezifischer Weise geändert wird. Zwischen HFA und HFR ließen sich an allen Alterspunkten und auch an den Tageszeitbereichen sehr enge Korrelationen nachweisen (Ergebnisse hier nicht weiter angeführt). Für HFA ( $p=0,002)$ und HFR $(p<0,001)$ konnte zwischen dem Alter von 40 LT und 15 LT eine Korrelation festgestellt werden. 
Abb. 4: Häufigkeitsverteilungen der Herzfrequenz (rechter Teil) und der Zeitdauer der Aktivitäts- und der Ruheperioden (linker Teil) von Milchrindkälbern an 4 Alterspunkten (Frequency distributions of heart rate (right part) and of the duration of activity and resting periods (left part) of dairy calves at four age points)
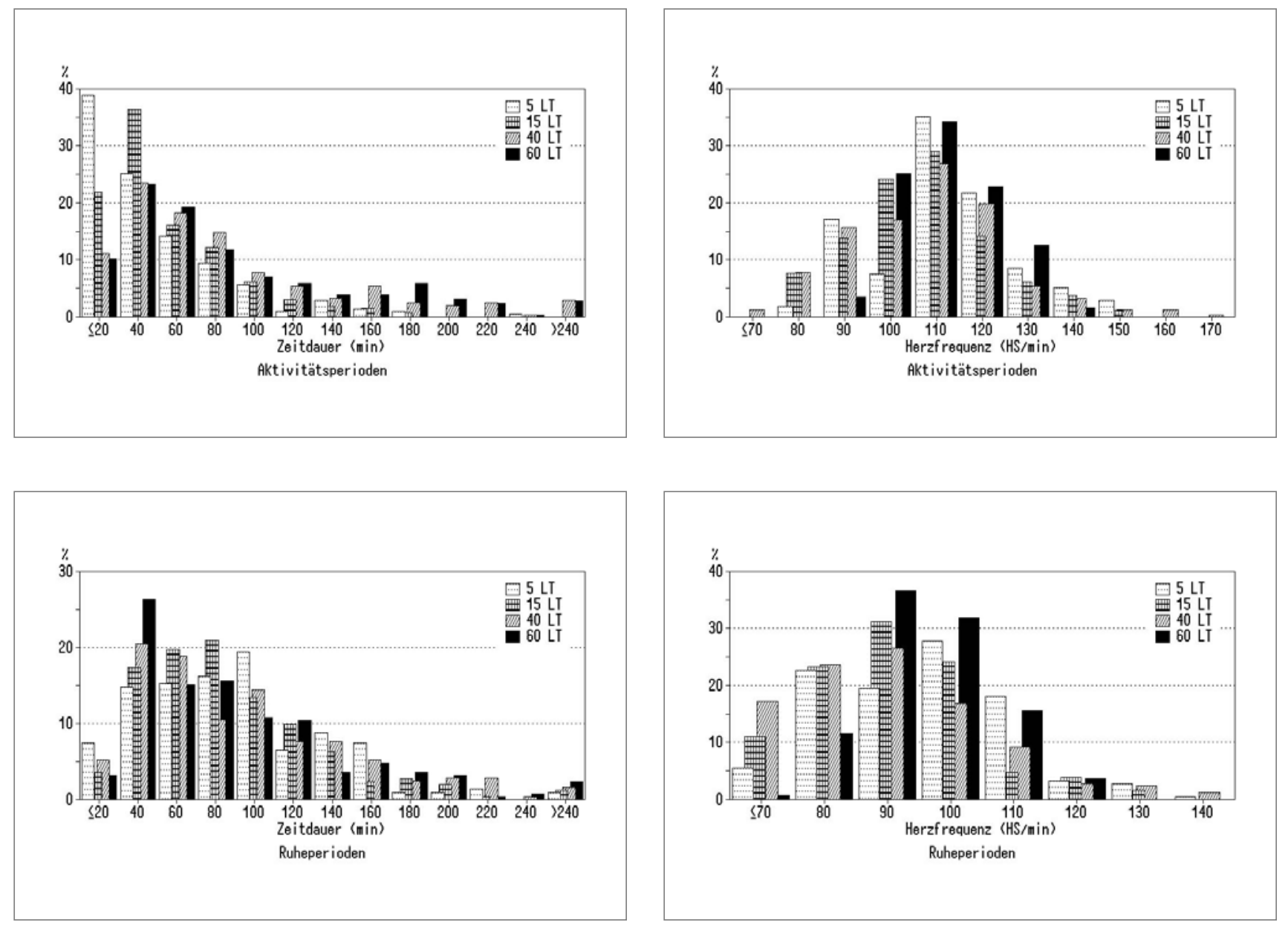

4. Diskussion

Rhythmen von HF, Blutdruck und Körpertemperatur, die mit den Wachzuständen (Arousal, Vigilanz) und Aktivitätsperioden der Tiere konform gehen (MATSUI u. SUGANO, 1989; SATO et al., 1995; YAMAYA et al., 1994), sind durch endogene Oscillatoren bedingt (MISTLBERGER, 1994 , Ratte; MOHR u. KRZYWANEK 1995, Schafe; SATO et al. 1995, Kaninchen und Ratten) und können durch exogene Stimulierungen (Licht, Temperatur, betriebliche Ereignisse in der Umwelt als Zeitgeber) synchronisiert werden. Aus den vorliegenden Befunden geht bei einem groben Raster der Messungen hervor, dass sich während der Aufzucht beim Übergang vom Winter in das Frühjahr bei den Kälbern ein circadianer Rhythmus der HF ausprägte und vorherrschend wurde. Auffällig waren Steigerungen von HF bei einem Anteil der Tiere in der Servicezeit an allen Alterspunkten (Abb. 1 bis 3).

Die während der Aktivitätsperioden erreichten Erregungsgrade und die damit verbundenen körperlichen Aktivitäten und Umsatzsteigerungen haben eine unterschiedliche Charakteristik bei Tieren in verschiedenen Altersbereichen und Haltungsvarianten sowie Anpassungs- und Funktionszuständen. Nahrungssuche, Art und Menge des aufgenommenen Futters und soziale Beziehungen spielen diesbezüglich eine größere Rolle, was zum Teil die qualitativ unterschiedlichen Beziehungen der HF zu ZDA, ZDR, 
GZA und GZR und zu dem Quotienten GZA/GZR erklärt. So konnten zwischen HF und dem Quotienten GZA/GZR bei 5 LT positive (HFA = 86,5 + 39,3GZA/GZR; $r$ = 0,538; $\mathrm{p}=0,017$; HFR $=68,8+41,3$ GZA/GZR, $r=0,567 ; \mathrm{p}=0,011$ ) und bei $60 \mathrm{LT}$ negative Beziehungen (HFA $=120,6-13,4$ GZA/GZR; $r=0,547 ; p=0,002$; HFR $=$ $98,8-8,1 \mathrm{GZA} / \mathrm{GZR}, \mathrm{r}=0,372 ; \mathrm{p}=0,047$ ) gefunden werden.

Enge negative Korrelationen und Regressionen zwischen GZA und GZR an den Alterspunkten sprechen für die Genauigkeit der Registrierung dieser Zeiten. Der zunehmende Quotient GZA/GZR von 0,63 auf 0,96 zwischen 15 LT und 40 LT, ein Ausdruck des Vorkommens längerer Aktivitätszeiten bei einigen Kälbern in diesem Alter (Abb. 1), größere inter- und intraindividuelle Variationen von ZDA und ZDR (Tab. 4) und auch des Quotienten GZA/GZR (Tab. 2) und die sich mit dem Alter ändernden Frequenzen von ZDA und ZDR in den Klassen 20, 40, 60, 80 und 100 min (Abb. 4) sind auf mehrere Einflußfaktoren (Entwicklungsqualität, typmäßige Reaktionsweise einerseits und Gruppenzusammensetzung, Nahrungsverfügbarkeit, Tierbetreuung und technisches Management, Lichtwirkungen andererseits) zurückzuführen, deren Effekte noch nicht ausreichend eingeschätzt werden können. Zunehmende ZDA und GZA bei Kälbern im Alter von 40 LT (Abb. 4, Tab. 2) sind auf Orientierungs- und Untersuchungsreaktionen, auf die vermehrte Aufnahme des in Raufen angebotenen Heues, der im Futtergang vorgelegten Silage und der im Kraftfutterautomaten verfügbaren Nahrung sowie auch auf soziale Interaktionen zurück zu führen. Wie an den Untersuchungsbefunden zu sehen ist (Abb. 1 und 3), war die circadiane Rhythmik der HF an den Alterspunkten unterschiedlich stark ausgeprägt. Das stärkere Hervortreten der circadianen Periodik gegen Ende der Milchernährungsperiode ist auch mit der vermehrten Festfutteraufnahme in kausalem Zusammenhang zu sehen.

In dieser Zeit sind individuelle Unterschiede und typmäßige Reaktionen der Kälber deutlicher erkennbar gewesen. Rhythmische Änderungen von ZDA und ZDR der Kälber an den Alterspunkten (Abb. 1, Tab. 3) waren nicht so deutlich wie z. B. bei Färsen während der Weidehaltung ausgeprägt (STEINHARDT u. THIELSCHER, 2000a), bei welchen stärkere soziale Synchronisierungseffekte vorhanden waren und der größte Anteil des Lichttages für die Nahrungssuche und -aufnahme genutzt werden mußte. Bei mutterlos aufgezogenen Milchrindkälbern (ERMGASSEN, 1996) und auch bei Saugkälbern der Mutterkuhhaltung (LEHR, 1997; STEINHARDT et al., 1997) hatte die Herzfrequenz (HF) eine große interindividuelle Variation und eine hohe Individualspezifität. Dies wird durch die vorliegenden Befunde bestätigt. Einflüsse von Geschlecht und Alter auf HF konnten an den Alterspunkten nachgewiesen werden. Die Stoffwechselintensität und die Wachstumsrate der Kälber, welche eine positive Beziehung zur HF haben, wiesen in der frühen Anpassungsperiode ebenfalls eine beträchtliche Variation auf (STEINHARDT und THIELSCHER, 2000b; STEINHARDT et al., 2000), wie auch an den vorliegenden Befunden zu sehen ist (Tab. 1). Die Verkleinerung der HF zwischen 5 und 15 LT (ERMGASSEN, 1996) war in der vorliegenden Untersuchung im Mittel geringgradig.

Während der Entwicklungsperiode mit überwiegender Ernährung der Kälber durch konzentrierte Flüssignahrung (Milch, MAT), die innerhalb kurzer Zeit aufgenommen werden kann, steht die übrige Tageszeit für die körperliche Ruhe oder für ein körperliches Training zur Verfügung, bei welchem motorische Koordinationen entwickelt werden und Lernprozesse erfolgen können (sogenanntes Spielverhalten). Ein höheres Niveau der HF war beim Stehen der Tiere gegenüber dem Liegen (Saugkälber der 
Mutterkuhhaltung: LEHR, 1997; STEINHARDT u. THIELSCHER, 2001a; Tränkkälber: STEINHARDT u. THIELSCHER, 2000c, 2001b) festzustellen. Die größeren Steigerungen der HF (HFA-HFR) im Tagesverlauf in Beziehung zu den MAT-Abrufzeiten und in der Servicezeit (Abb. 2) sind ein Ausdruck gesteigerter sympathischer nervaler Aktivität in Verbindung mit der Nahrungsaufnahme bei Jungtieren (BLOOM et al., 1975; BOWMAN et al. 1997, ERMGASSEN, 1996) und der lokomotorischen Aktivität (Laufen, Springen). Bei Saugkälbern der Mutterkuhhaltung (Winterstallhaltung), bei denen eine kaum eingeschränkte Nahrungsverfügbarkeit und damit eine ad-libitum-Fütterung gegeben war, waren die Nahrungsaufnahme und ein individuell unterschiedlich ausgeprägtes Spielverhalten mit einer infradianen Periodik der HF verbunden (STEINHARDT und THIELSCHER, 2001a). Erregungs- und Aktivitätssteigerungen schienen auch bei mutterloser Aufzucht und Einzeltierhaltung während der Tränkezeit und danach vorzukommen und mit einer wenn auch beschränkten motorischen Aktivität verbunden gewesen zu sein, denn ZDA und ZDR und auch GZA und GZR unterschieden sich an den Alterspunkten zwischen Kälbern in Einzeltier- und Gruppenaufstallung bei limitierter Verfügbarkeit der Flüssignahrung nicht signifikant (STEINHARDT und THIELSCHER, 2002, STEINHARDT et al., 2002).

Die mit dem Tränken verbundenen Aktivitäten der Tiere im Tränkestand und die Geräusche können in Gruppenhaltungen als Zeitgeber wirken, in Abhängigkeit von Alter und Entwicklungsqualität der Kälber eine Aktivierung eines größeren Anteils der Tiere und damit Synchronisierungseffekte zur Folge haben und auch das Spielverhalten der Tiere überlagern. Bei 4 Tränkeabrufterminen waren 3 Aktivitätsphasen und ein hohes Aktivitätsniveau zwischen 08.00 und 12.00 Uhr (Servicezeit 09.00 bis 12.00 Uhr), zwischen 14.00 und 18.00 Uhr sowie zwischen 20.00 und 23.00 Uhr festgestellt worden (ZERBE, 1998). „Stärkere Tiere“ verweilten infolge der ständigen „Eigenbelohnungen“ häufiger und länger im Tränkestand und erwarben einen höheren Anspruch an „Spielsaugen“ (ZERBE, 1998). Einflüsse des Alters der Kälber, der eingeschränkten Nahrungsverfügbarkeit und der Jahreszeit sind in der genannten Untersuchung anscheinend nicht genauer analysiert worden. Die sogenannten Kontrollbesuche (Saugen ohne Milchabruf) waren an allen Abrufzeitpunkten häufiger als die Besuche mit Milchabruf, sie waren überwiegend vor diesen zu beobachten, und ihre Häufigkeit nahm im Verlaufe des Tages an den Abrufzeitpunkten zu (ZERBE, 1998). Höhere Anteile der ZDA in den Klassen 20 und 40 min bei Kälbern im Alter von 5 LT und 15 LT deuten auf den häufigeren Wechsel zwischen Ruhe und Aktivität hin (Abb. 4). ZDR von 40, 60, 80 und sogar $100 \mathrm{~min}$ an allen Alterspunkten und mit dem Alter der Kälber zunehmende Anteile der ZDR von 40 min Dauer (Abb. 4) können bei Berücksichtigung größerer Entwicklungsunterschiede der Tiere kausal auch in Verbindung mit Ermüdungserscheinungen und physischer Erschöpfung einzelner Tiere eingeschätzt werden. Dazu wären weitere Untersuchungen notwendig.

Die Hauptkomponenten des Spielverhaltens wie lokomotorisches Spielen in Gruppen, auch paralleles Spielen ohne physischen Kontakt genannt, spielerisches Kämpfen von zwei oder mehr Kälbern, spielerisches Aufspringen und Bodenspiel (BYERS, 1984) haben unterschiedliche Zeitanteile im Verlaufe der Aufzucht. Während das lokomotorische Spielen in Gruppen mehr bei jüngeren Kälbern zu beobachten ist, nehmen spielerisches Kämpfen, Aufspringen und Bodenspiel bei älteren Kälbern zu. Der Grad des lokomotorischen Spielens und dessen Synchronie verringerten sich über die Wochen bei mutterlos aufgezogenen Kälbern (Fütterung 2 mal pro Tag aus Eimern), das spiele- 
rische Kämpfen, in welches männliche Tiere mehr einbezogen waren, jedoch nicht (JENSEN et al., 1998; JENSEN und KYHN, 2000). Angaben über das Spielverhalten der Kälber bei beständig wechselnder Gruppenzusammensetzung und über dessen gehäuftes Vorkommen an spezifischen Zeitpunkten im Tagesverlauf oder in Abhängigkeit von anderen Verhaltensweisen wie Nahrungsaufnahme und Körperschutzreaktionen sind kaum vorhanden.

Die mit Hilfe der kontinuierlichen Herzfrequenzmessung bei Milchrindkälbern an verschiedenen Alterspunkten vorgenommene Charakterisierung der Herzfrequenzkennwerte in Tageszeitbereichen mit gesteigerter körperlicher Aktivität und in solchen mit relativer körperlicher Ruhe ermöglichen eine orientierende Einschätzung der Rhythmizität von Funktionsabläufen unter spezifischen Haltungsbedingungen und auch von deren Änderungen infolge des Wachstums und der Entwicklung sowie der Anpassung der Kälber an die Haltungsbedingungen in den Jahreszeiten. Intensivere Untersuchungen an einzelnen Kälbern im Verlaufe der Aufzuchtperiode wären notwendig, um die Beziehungen der Rhythmizität physiologischer Variablen mit den Nutzleistungskriterien und die typmäßige Reaktionsweise genauer untersuchen zu können.

\section{Literatur}

BLOOM, S.R.; EDWARDS, A.V.; HARDY, R.N.; MALINOWSKA, K.W.; SILVER, M.:

Cardiovascular and endocrine responses to feeding in the young calf. J. Physiol. Lond. 253 (1975), 135155

BOWMAN, E.C.J.; RODERICK, G.P.; BLOOM, S.R.; EDWARDS, A.V.:

Role of adrenoceptors in the hypertensive response to feeding in the conscious calf. Am. J. Physiol. 272 (Regulatory Integrative Comp. Physiol. 41) (1997), R607-R614

BYERS, J.A.:

Play in ungulates. In: SMITH, P. K. (ed.): Play in Animals and Humans. Basil Blackwell Publ. Lm. Pt. 1 (1984), 43-65

ERMGASSEN, K.:

Untersuchungen zur Herzfrequenz und zu klinischen Vitalitätsparametern bei Kälbern in Beziehung zu Tragzeit, Geburtsverlauf, Geschlecht und Rasse. Univ. Leipzig, Vet. med. Diss., 1996

JENSEN, M.B.; KYHN, R.:

Play behaviour in group-housed dairy calves, the effect of space allowance. Appl. Anim. Behav. Sci. 67(2000), 35-46

JENSEN, M.B.; VESTERGAARD, K.S.; KROHN, C.C.:

Play behaviour in domestic calves kept in pens: the effect of social contact and space allowance. Appl. Anim. Behav. Sci. 56 (1998), 97-108

LEHR, A.:

Verhaltensphysiologische Reaktionen bei Mutterkühen und ihren Kälbern im peripartalen Zeitraum. 3. Trenthorster Kolloquium 5. und 6. Dezember 1996. Landbauforschung Völkenrode, Sonderheft 177 (1997), 138-148

MATSUI, K.; SUGANO, S.:

Relation of intrinsic heart rate and autonomic nervous tone to resting heart rate in the young and the adult of various domestic animals. - Jpn. J. Vet. Sci. 51 (1989), 29-34

MISTLBERGER, R.E.:

Circadian food-anticipatory activity: formal models and physiological mechanisms. Neuroscience and Biobehavioural Rev. 18 (1994) 2, 171-195

MOHR, E.G.; KRZYWANEK, H.:

Endogenous oscillator and regulatory mechanisms of body temperature in sheep. Physiol. Behav. 57 (1995), 339-347

SATO, K.; CHATANI, F.; SATO, S.:

Circadian and short-term variabilities in blood pressure and heart rate measured by telemetry in rabbits and rats. J. Autonom. Nerv. Syst. 54 (1995), 234-246

STEINHARDT, M.; THIELSCHER, H.-H.; ERMGASSEN, K.; LEHR, A.: 
Langzeitmessungen in entwicklungs- und verhaltensphysiologischen Untersuchungen bei landwirtschaftlichen Nutztieren am Beispiel der Herzschlagfrequenz. Schriftenreihe des Forschungsinstitutes für die Biologie landwirtschaftlicher Nutztiere (FBN), Heft 9 (1997), 47-70

STEINHARDT, M.; THIELSCHER, H.-H.:

Herzfrequenzkennwerte, Aktivitätsperioden und Blutmeßwerte trächtiger, nicht laktierender Rinder bei Weidehaltung. Tierärztliche Umschau 55 (2000a) 3, 133-140

STEINHARDT, M.; THIELSCHER, H.-H.:

Wachstum und Entwicklungsqualität von Milchrindkälbern in Gruppenhaltung mit Tränkeautomatenfütterung. Physiologische Variablen und deren Änderungen in spezifischen Altersperioden. Arch. Tierz., Dummerstorf 43 (2000b) 1, 27-44

STEINHARDT, M.; THIELSCHER, H.-H.:

Aktivitätsperioden und Rhythmizität physiologischer Variablen von am Tränkeautomaten aufgezogenen Milchrindkälbern. Herzfrequenz, Aktivitäts- und Ruheperioden bei männlichen und weiblichen Tieren an verschiedenen Alterspunkten. Landbauforschung Völkenrode 50 (2000c) 3/4, 163-178

STEINHARDT, M.; THIELSCHER, H.-H.; GRÜNBERG, W.:

Tiergerechte Haltung und physiologische Funktionen von Tieren. Entwicklungsqualität und Anpassung von Saugkälbern der Mutterkuhhaltung und von in Gruppen am Tränkeautomaten aufgezogenen Kälbern der Deutschen Rotbunten. Landbauforschung Völkenrode 50 (2000) 3/4, 181-198

STEINHARDT, M.:

Aktivitätsperioden und Rhythmizität physiologischer Variablen von Saugkälbern der Mutterkuhhaltung. Herzfrequenz, Aktivitäts- und Ruhezeiten während der Anpassung der Tiere an die Weidehaltung. Landbauforschung Völkenrode 51 (2001) 3, 109-119

STEINHARDT, M.; THIELSCHER, H.-H.:

Herzfrequenz und Tagesperiodik bei Saugkälbern der Mutterkuhhaltung an verschiedenen Alterspunkten. Tierärztl. Umschau 56 (2001a), 206-215

STEINHARDT, M.; THIELSCHER, H.-H.:

Herzfrequenz und Tagesperiodik bei Milchrindkälbern während der Aufzucht am Tränkeautomaten. Tierärztl. Umschau 56 (2001b), 413 - 423

STEINHARDT, M.; THIELSCHER, H.-H.:

Tiergerechte Haltung und physiologische Funktionen von Tieren. Effekte der Haltungsbedingungen auf die Aktivitätsperioden und Rhythmizität physiologischer Variablen von Milchrindkälbern. Herzfrequenz, Aktivitäts- und Ruhezeiten der Kälber an verschiedenen Alterspunkten. Landbauforschung Völkenrode 52 (2002) (im Druck)

STEINHARDT, M.; THIELSCHER, H.-H.; NIEMANN, H.:

Identische Zwillinge bei der Untersuchung des Einflusses von Haltungsbedingungen auf landwirtschaftliche Nutztiere. Herzfrequenz, Aktivitäts- und Ruhezeiten von Zwillingskälbern in zwei Haltungsvarianten. Tierärztl. Umschau 57 (2002) (im Druck)

YAMAYA, Y.; KUBO, K.; AMADA, A.:

Diurnal rhythms of R-R interval and R-R interval variability in the young thoroughbred horse. J. Equine Sci. 5 (1994), 83-86

ZERBE, F.:

Einsatz von Tränkeautomaten in der Gruppenhaltung von Aufzuchtkälbern unter besonderer Berücksichtigung des Saug- und Futteraufnahmeverhaltens. Univ. Hannover, Vet. Med. Diss., 1998

Eingegangen: 04.01.2002

Akzeptiert: 10.06.2002

Anschrift der Verfasser

Dr. habil. MARTIN STEINHARDT, Dr. HANS - HERMANN THIELSCHER

Institut für Tierzucht und Tierverhalten - FAL

Trenthorst/Wulmenau

D-23847 Westerau 